\title{
Subbands in the doped two-orbital Kanamori-Hubbard model
}

\author{
K. Hallberg $\odot^{*}$ and Y. Núñez-Fernández $\odot$ \\ Centro Atómico Bariloche and Instituto Balseiro, CNEA and CONICET, 8400 Bariloche, Argentina
}

(Received 3 June 2020; revised 31 August 2020; accepted 10 December 2020; published 23 December 2020)

\begin{abstract}
We calculate and resolve with unprecedented detail the local density of states (DOS) and momentumdependent spectral functions at zero temperature of one of the key models for strongly correlated electron materials, the degenerate two-orbital Kanamori-Hubbard model, by means of the dynamical mean-field theory, which uses the density matrix renormalization group as the impurity solver. When the system is hole doped and in the presence of a finite interorbital Coulomb interaction, we find the emergence of a novel holon-doublon in-gap subband which is split by the Hund's coupling. We also observe interesting features in the DOS, such as the splitting of the lower Hubbard band into a coherent narrowly dispersing peak around the Fermi energy, and another subband which evolves with the chemical potential. We characterize the main transitions giving rise to each subband by calculating the response functions of specific projected operators and by comparing with the energies in the atomic limit. The detailed results for the spectral functions found in this work pave the way to study with great precision the microscopic quantum behavior in correlated materials.
\end{abstract}

DOI: 10.1103/PhysRevB.102.245138

\section{INTRODUCTION}

Materials with strong electron-electron correlations due to interactions in local orbitals, like transition-metal oxides with partially filled $d$ or $f$ shells, are among the most interesting problems in condensed matter physics. Their fascinating properties, such as high-temperature superconductivity, colossal magnetoresistance, correlation-driven metal-insulator transitions, or heavy-fermion behavior, and their sensitivity to external fields make them attractive candidates for applications. However, in spite of important progress made in analytical and numerical tools, it is still difficult to obtain precise and detailed theoretical electronic structure results to compare with experiments, like angular-resolved photoemission, inverse photoemission experiments, or optical conductivity measurements.

Some recent theoretical studies used the dynamical meanfield theory (DMFT) [1-3] to analyze multiplets in photoemission experiments in chalcogenides and pnictides [4], the role of atomic states in interacting intermediate valence systems [5], the electronic structure of heavy-fermion compounds [6] and pnictides [7], and the competition of different interactions, like the local Coulomb of Hund interactions in multiorbital models [8].

The development of more sophisticated numerical methods to solve the effective impurity solver of the DMFT, such as using the density matrix renormalization group, (DMFT+DMRG) [9-11], and later developments [12-14] allowed for the calculation of spectral densities with high precision. The DMFT+DMRG was used to study the halffilled multiorbital Kanamori-Hubbard model (KHM), which includes intra- and interorbital Coulomb interactions ( $U$ and

*karen@cab.cnea.gov.ar
$\left.U_{2}\right)$ as well as a ferromagnetic Hund coupling $(J)$ between the orbitals. In particular, for the two-orbital model on the Bethe lattice, well-defined quasiparticle peaks were observed in the local density of states (DOS) for the half-filled system in the quasilocalized metallic state close to the Mott transition and in the orbital selective phases (OSP). These peaks were characterized as formed by interorbital holon-doublon bound states [15]. Subsequent papers confirmed their existence using other methods, like slave particles [16], extensive numerical calculations for the three-orbital model also within the DMFT using the numerical renormalization group (NRG) as the impurity solver [17], or using exact diagonalization to obtain their splitting with $J$ in the OSP [18]. Photoinduced nonequilibrium holon-doublon excitations have been also obtained in a one-dimensional two-orbital version of the model studied here [19], where they were attributed exclusively to the Hund interaction. The structure in the inner edges of the Hubbard bands in the half-filled Hubbard model close to the metal-insulator transition $[12,20]$ has also been characterized as holon-doublon excitations [21], albeit between nearestneighbor sites.

In this work we revisit the two-band KHM $[22,23]$. Using the DMFT+DMRG numerical technique, we obtain the single-particle density of states for the arbitrarily doped case and for an ample range of parameters. We observe a much richer structure than obtained in previous calculations, including the existence of in-gap subbands, which we characterize by calculating the response functions of specific operators and also by comparing to the atomic limit. In particular, we find that one of the subbands is formed mainly by interorbital holon-doublon excitations which, for high enough ratios of $U_{2} / U$, emerge and separate from the upper Hubbard band (UHB). The doped lower Hubbard band (LHB) also splits into subbands composed mainly by well-characterized excitations. These results show that there exists a much more interesting 


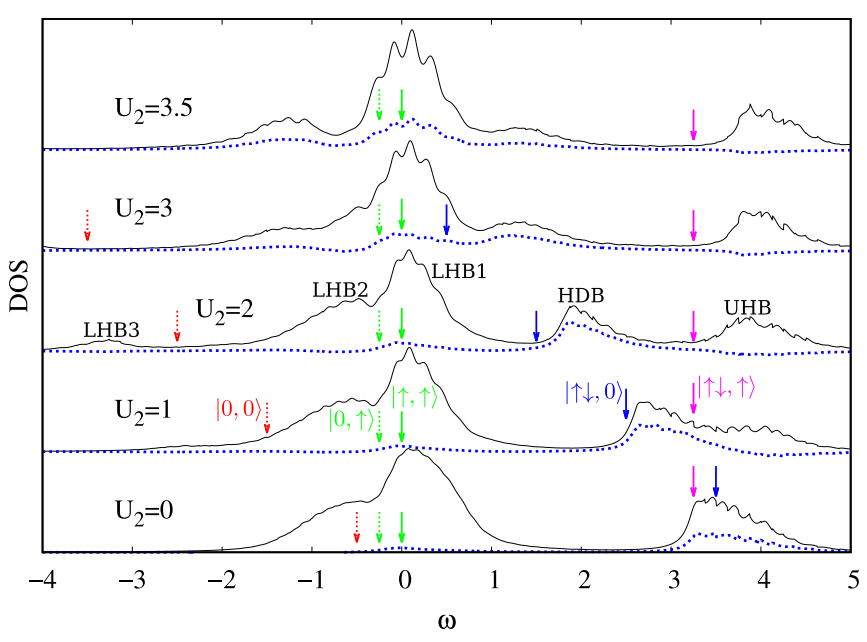

FIG. 1. Single-particle densities of states (DOS) for the twoorbital KHM for fixed $U=3.5, J=0$, and $\mu=-1.5$ with varying $U_{2}$. The arrows indicate the atomic limit energies. Also shown is $A_{H D}(\omega)$ (blue dotted line).

scenario of excitations than previously thought of in these paradigmatic models for correlated systems.

The Kanamori-Hubbard model reads

$$
H=\sum_{\langle i j\rangle \alpha \sigma} t_{\alpha} c_{i \alpha \sigma}^{\dagger} c_{j \alpha \sigma}-(\mu-\epsilon) \sum_{i} n_{i}+\sum_{i} \hat{V}_{i},
$$

where $\langle i j\rangle$ are nearest-neighbor sites in the Bethe lattice, $\alpha=1,2$ are orbital indices, $\sigma$ the spin index, and $c$ and $n_{i}=$ $\sum_{\alpha \sigma} c_{i \alpha \sigma}^{\dagger} c_{i \alpha \sigma}$ the usual electron destruction and number operators. We consider $t_{1}=t_{2}=0.5$, no interorbital hybridization, and $\epsilon=-U / 2-U_{2}+J / 2$ such that at $\mu=0$ the system is half filled.

The on-site interactions $\hat{V}_{i}$ are

$$
\begin{aligned}
\hat{V}_{i}= & U \sum_{\alpha} n_{i \alpha \uparrow} n_{i \alpha \downarrow}+\sum_{\sigma \sigma^{\prime}}\left(U_{2}-J \delta_{\sigma \sigma^{\prime}}\right) n_{i 1 \sigma} n_{i 2 \sigma^{\prime}} \\
& -J\left(c_{i 1 \uparrow}^{\dagger} c_{i 1 \downarrow} c_{i 2 \downarrow}^{\dagger} c_{i 2 \uparrow}+c_{i 1 \downarrow}^{\dagger} c_{i 1 \uparrow} c_{i 2 \uparrow}^{\dagger} c_{i 2 \downarrow}\right) \\
& -J\left(c_{i 1 \uparrow}^{\dagger} c_{i 1 \downarrow}^{\dagger} c_{i 2 \uparrow} c_{i 2 \downarrow}+c_{i 2 \uparrow}^{\dagger} c_{i 2 \downarrow}^{\dagger} c_{i 1 \uparrow} c_{i 1 \downarrow}\right) .
\end{aligned}
$$

We apply the DMFT [1-3] to solve the model using the DMRG [24,25] to obtain the impurity's Green's functions [9-11] on the real axis with a small imaginary offset $\eta \sim 0.1$ using $L=48$ spinfull orbitals.

\section{RESULTS}

First we present results for $J=0$ while varying $U_{2}$ (i.e., not necessarily in the rotational invariant case). In all figures we show the results for one of the orbitals since, by symmetry, both orbitals are equivalent.

In Fig. 1 we show the sequence of local single-particle DOS for fixed on-site $U=3.5$ and $\mu=-1.5$ with varying $U_{2}$. For $U_{2}=0$ we have the two well-known UHB and LHB. For this chemical potential the system is slightly hole doped with a finite DOS at the Fermi energy. When $U_{2}$ increases, we can see a subband separating and emerging from the UHB towards lower energies and which evolves into an independent well-defined subband located within the Mott-Hubbard gap for large enough $U_{2}$. As we will show below, we identify this
TABLE I. Relevant representative atomic configurations and their energy relative to the energy of the atomic state $|\uparrow, \uparrow\rangle$.

\begin{tabular}{lc}
\hline \hline Representative atomic configuration $\left|s_{1}, s_{2}\right\rangle$ & $\epsilon_{\left|s_{1}, s_{2}\right\rangle}$ \\
\hline$|0,0\rangle$ & $U+U_{2}+2 \mu$ \\
$|\uparrow, 0\rangle$ & $U / 2+J / 2+\mu$ \\
$|\uparrow, \uparrow\rangle$ & 0 \\
$|\uparrow, \downarrow\rangle \pm|\downarrow, \uparrow\rangle$ & $J \mp J$ \\
$|0, \uparrow \downarrow\rangle \pm|\downarrow \uparrow, 0\rangle$ & $U-U_{2}+J \mp J$ \\
$|\uparrow \downarrow, \uparrow\rangle$ & $U / 2+J / 2-\mu$ \\
\hline \hline
\end{tabular}

subband as formed mainly by local interorbital holon-doublon pairs: a holon-doublon band (HDB). The approximate location of this band in the atomic limit is given by the expressions in Table I and indicated by blue arrows in the figure. The HDB continues to move to lower energies with $U_{2}$ until it overlaps with the LHB. When $U_{2}=U=3.5$ and $J=0$ the system has SU(4) rotational symmetry and the holon-doublon (HD) excitations are degenerate with the $\left|\sigma, \sigma^{\prime}\right\rangle$ states (see Table I). The HDB emerging in the hole-doped situation is an incoherent subband which has a different character than the narrow HD quasiparticle peak stemming from the coherent metallic DOS in the half-filled case [15].

It is interesting to see that the LHB splits into three subbands with $U_{2}$. Close to the Fermi energy we find a splitting into two subbands: one corresponding to a band centered at $\omega=0$ (LHB1) and another subband, (LHB2), separated from the first one by a minigap for large $U_{2}$. In addition, there is a small feature at larger negative energies (LHB3) which also moves with $U_{2}$. The UHB transfers part of its weight to the HDB.

In Fig. 2 we plot the DOS for particular values of $U$ and $U_{2}$ while varying the chemical potential $\mu$. For the half-filled case $(\mu=0)$ the system is insulating for these local interaction parameters. As soon as the system is doped with $\mu$, we find an in-gap structure appearing in between these Hubbard

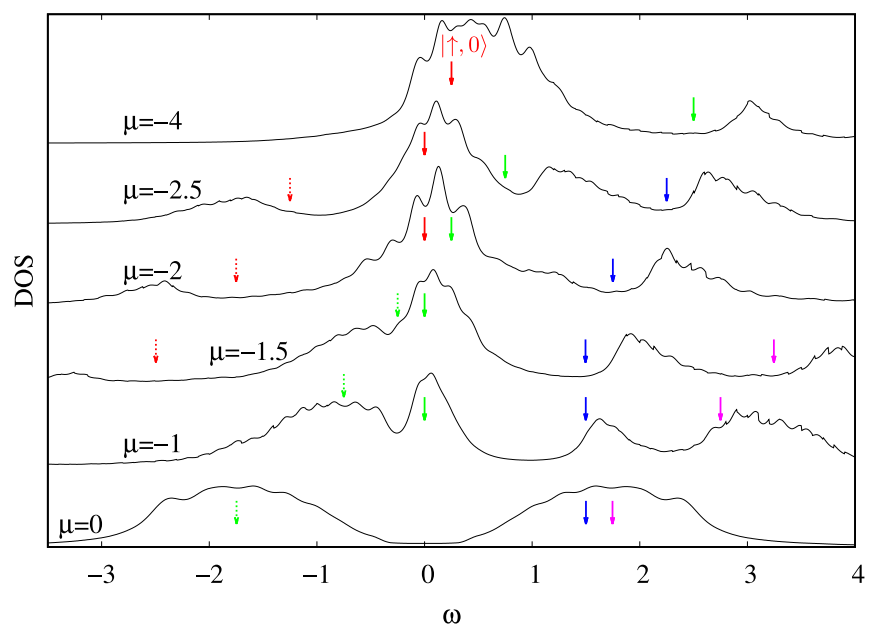

FIG. 2. DOS for fixed on-site $U=3.5, J=0$, and $U_{2}=2$ vs $\mu$, from the half-filled case $(\mu=0, \eta=0.2)$ to the extremely doped case $\mu=-4$. The arrows (atomic limit energies) have the same color code as in Fig. 1. 


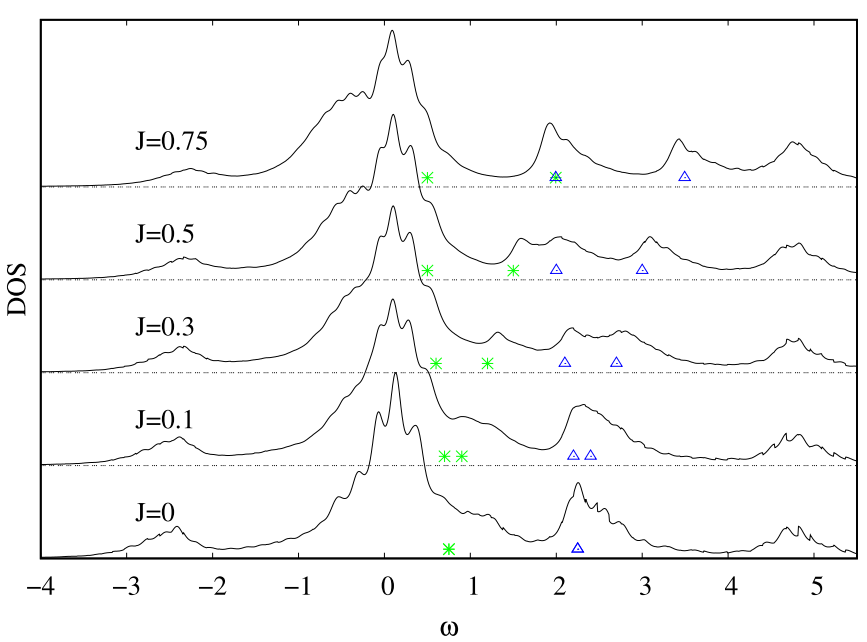

FIG. 3. DOS vs Hund coupling $J$ for fixed $U=3.5, U_{2}=2$, and $\mu=-2$. The $\mathrm{SU}(4)$ orbitally symmetric case is at $J=0.75$. The splitting of $2 J$ of the HDB and the $|\uparrow, \downarrow\rangle \pm|\downarrow, \uparrow\rangle$ bands is marked as a guide by blue triangles and by green asterisks, respectively.

bands which we identify as the HDB mentioned before and which is always present as long as the bands are metallic. The approximate location of this band in the atomic limit is, again, given by the expressions in Table I and are indicated by blue arrows in the figure.

Here we also observe the splitting of the LHB into three subbands, the low-energy ones being LHB1 (around $\omega=0$ ) and LHB2. The LHB2 subband crosses the Fermi energy when decreasing $\mu$ (i.e., doping with holes) and continues moving to higher energies with hole doping (green arrows). The LHB3 is also formed at larger negative energies due to $U_{2}$ (red arrows).

We now consider a finite Hund interaction $J$. In this case we find a much richer structure: the HDB and the LHB2 bands split into two bands separated by $2 J$ (see Fig. 3), the former due to the pair-hopping term and the latter due to the spin-flip term in Eq. (2). For the rotationally symmetric case in which $U_{2}=U-2 J$, the lower energy states of the HDB coincides with the higher energy ones of the LHB2 (see Table I). Splittings due to the Hund's $J$ were also found in Ref. [18] but for the half-filled orbital selective Mott phase (OSMP). We show here that it is not essential to be in an OSMP nor to have a small ratio of hoppings in both bands to observe the HDB or its splittings due to $J$ as stated in that work. We also expect that splittings with $J$ should be observed in the model studied in [17], however, this is not reported in that work.

A gross estimation of the energies of these novel excitations can be done by simple calculations considering the eigenstates of the two-orbital atomic limit represented as $\left|s_{1}, s_{2}\right\rangle$ where $s_{1}, s_{2} \in\{0, \uparrow, \downarrow, \uparrow \downarrow\}$, see Table I. The relative energy $\epsilon_{\left|s_{1}, s_{2}\right\rangle}$ of each final configuration $\left|s_{1}, s_{2}\right\rangle$ with respect to the energy $\epsilon_{0}$ of the atomic ground state is represented in the DOS at frequency $\omega=\epsilon_{\left|s_{1}, s_{2}\right\rangle}-\epsilon_{0}\left(\omega=-\epsilon_{\left|s_{1}, s_{2}\right\rangle}+\epsilon_{0}\right)$ for creating (destroying) an electron and is indicated with colored arrows in the figures. The ground state for the half-filled or lightly doped case is $|\uparrow, \uparrow\rangle$, while for higher dopings there

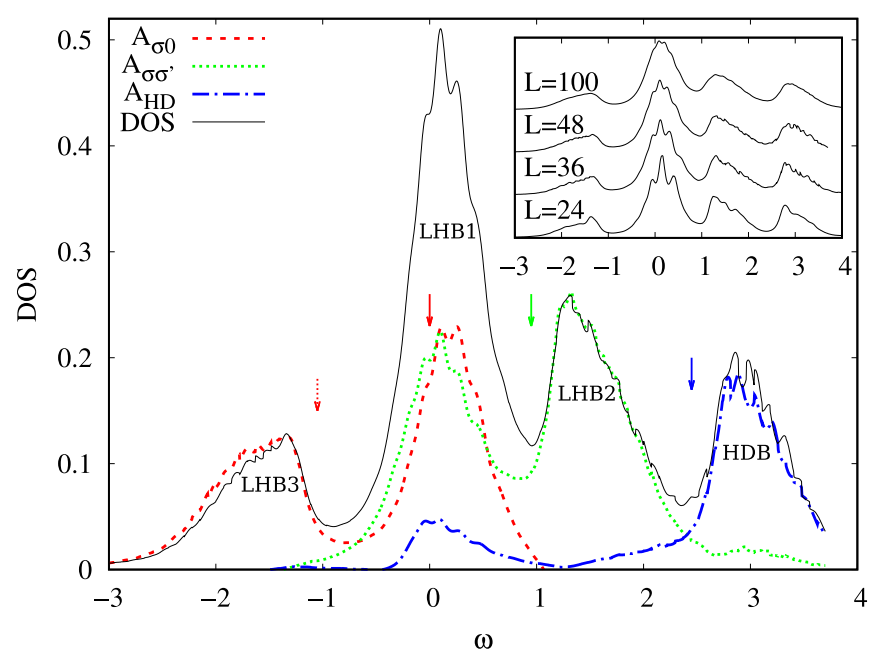

FIG. 4. Decomposition of the DOS for $U=3.5, U_{2}=2, J=0$, $\mu=-2.7$ onto the projected excitations $A_{s_{1}, s_{2}}(\omega)$ defined in Eqs. (3) and (4). The UHB is out of scale. Clearly resolved are the HDB and the threefold split LHB subbands (arrows represent the atomic energies, same color code as Fig. 1). The inset shows the scaling for different bath lengths using $\eta=0.1$.

is a level crossing (cf. Fig. 2) and the atomic ground state is $|\uparrow, 0\rangle$ so the excitation energies must be rescaled accordingly.

To characterize the excitations, we calculate the dynamical response function for creating or destroying an electron on each of the main components of the ground state according to Table I. Generalizing the operators defined in Ref. [21] allows us to calculate directly the local character of the excitations without having to resort to approximations: we define the Green's functions $A_{s_{1}, s_{2}}(\omega)=A_{s_{1}, s_{2}}^{>}(\omega)+A_{s_{1}, s_{2}}^{<}(-\omega)$ with

$$
\begin{aligned}
& A_{s_{1}, s_{2}}^{>}(\omega)=-\frac{1}{\pi} \mathfrak{s}\left\langle c_{1 \uparrow}\left(\omega+i \eta-H_{i m p}+E_{0}\right)^{-1} X_{s_{1}, s_{2}}^{\dagger}\right\rangle, \\
& A_{s_{1}, s_{2}}^{<}(\omega)=-\frac{1}{\pi} \mathfrak{s}\left\langle c_{1 \uparrow}^{\dagger}\left(\omega+i \eta-H_{i m p}+E_{0}\right)^{-1} X_{s_{1}, s_{2}}\right\rangle,
\end{aligned}
$$

where the expectation is taken for the ground state with energy $E_{0}$ of the DMFT Hamiltonian $H_{i m p}$. The excitations are $X_{s_{1}, s_{2}}^{\dagger}=P_{s_{1}, s_{2}} c_{1 \uparrow}^{\dagger}$, and their reverse action $X_{s_{1}, s_{2}}=$ $c_{1 \uparrow} P_{s_{1}, s_{2}}$. The projector $P_{s_{1}, s_{2}}=\left|s_{1}, s_{2}\right\rangle\left\langle s_{1}, s_{2}\right|$ is used to select the corresponding atomic configuration $\left|s_{1}, s_{2}\right\rangle$. Note that adding all possible configurations gives the total DOS, since $\sum_{s_{1}, s_{2}} P_{s_{1}, s_{2}}=1$. We are particularly concerned about the following excitations (and their reverse actions) for orbital 1:

(i) HD states $\quad(|\downarrow, 0\rangle \rightarrow|\uparrow \downarrow, 0\rangle): \quad X_{\uparrow \downarrow, 0}^{\dagger}=n_{1 \downarrow}(1-$ $\left.n_{2 \uparrow}\right)\left(1-n_{2 \downarrow}\right) c_{1 \uparrow}^{\dagger}$

(ii) $|0, \uparrow\rangle \rightarrow|\uparrow, \uparrow\rangle: X_{\uparrow, \uparrow}^{\dagger}=n_{2 \uparrow}\left(1-n_{2 \downarrow}\right)\left(1-n_{1 \downarrow}\right) c_{1 \uparrow}^{\dagger}$

(iii) $\quad|0,0\rangle \rightarrow|\uparrow, 0\rangle: \quad X_{\uparrow, 0}^{\dagger}=\left(1-n_{1 \downarrow}\right)\left(1-n_{2 \uparrow}\right)(1-$ $\left.n_{2 \downarrow}\right) c_{1 \uparrow}^{\dagger}$

In Fig. 4 we show a breakdown of the main single-particle excitations and a comparison to the total DOS. We observe that the HDB is formed mainly by holon-doublon states of the form $|\uparrow \downarrow, 0\rangle$. We find that the LHB3 is formed by transitions 


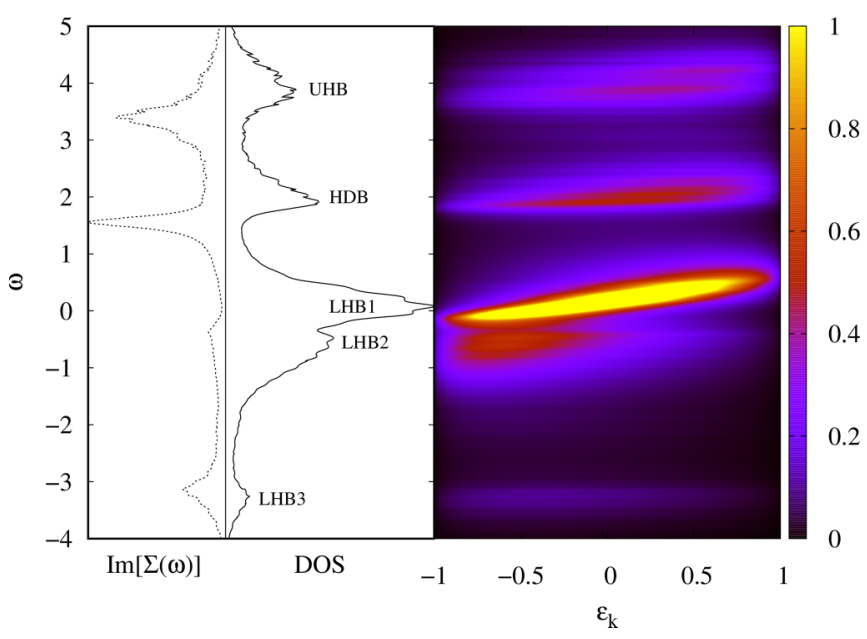

FIG. 5. Spectral function $A(k, \omega)$ for $U=3.5, U_{2}=2, J=0$, and $\mu=-1.5$. The band around $\omega=0$ disperses across the Fermi energy. The energy gaps separating each subband are apparent and marked by peaks in the imaginary part of the self-energy (also plotted).

$|0,0\rangle \leftrightarrow|\uparrow, 0\rangle$ given by $A_{\sigma 0}(\omega)$, while the LHB2 is formed by the transitions $|0, \uparrow\rangle \leftrightarrow|\uparrow, \uparrow\rangle$ given by $A_{\sigma \sigma^{\prime}}(\omega)$ (for all spin configurations). Instead, the DOS around the Fermi energy contains an admixture of both excitations plus a smaller weight of HD states. These subbands can be also clearly recognized in Figs. 1, 2, 3, and 5. For small chemical potentials, the LHB2 subband generated by $A_{\sigma \sigma^{\prime}}$ appears below the Fermi energy (see Fig. 1) and crosses $\omega=0$ with hole doping (see Fig. 2). In the inset we show the scaling with number of bath sites, where the subband structure is maintained.

We have also calculated the momentum-resolved spectral function $A(k, \omega)$ where within the DMFT the momentum enters via the noninteracting dispersion relation $\epsilon_{k}$ [1] (see Fig. 5). We find that the lowest-lying states around zero (LHB1) disperse across the Fermi energy and are separated by a minigap from the LHB2 (in this case at $\omega<0$ ) which has a large weight for negative momenta. Also seen are the LHB3 subband at $\omega \sim-3.5$ and the UHB at $\omega \sim 4$.

\section{CONCLUSIONS}

In this work we revisit the doped two-orbital KanamoriHubbard model, a key model to study multiband correlated materials. Using the high-precision DMFT+DMRG technique we find novel excitations which have passed unnoticed in numerous previous studies,

We observe clear subbands within the Mott gap formed mainly by holon-doublon pairs which are pulled down from the upper Hubbard band to lower energies by the interorbital Coulomb interaction $U_{2}$ and are split by the magnetic Hund's interactions by $2 J$. The lower Hubbard band also splits into three subbands with $U_{2}$, two of which lie close to the Fermi energy: One is the metallic peak at around $\omega=0$ while the other one involves states of the form $|s 1, s 2\rangle=\left|\sigma, \sigma^{\prime}\right\rangle$ in the atomic limit, whose energy increases with $U_{2}$ and crosses the Fermi energy with $\mu$. We have also characterized each subband by a careful comparison with the states in the atomic limit and by calculating projected response functions.

The main qualitative results presented here do not depend on particular choices of parameters. We expect these subbands to be robust with a small interorbital hybridization, also for the case of three or more orbitals and for other types of lattices or even in one dimension. We also expect similar features to exist for other dopings and related models. We hope that the results presented here together with the possibility of calculating more precise spectral functions for models of correlated materials will stimulate a closer study of the details of experimental results and hence contribute to unveil the complex and elusive microscopic behavior of strongly correlated materials.

\section{ACKNOWLEDGMENTS}

We acknowledge support from Projects No. PICT 20160402 and No. 2018-01546 (ANPCyT) and PIP2015 11220150100538 CO (CONICET). We thank G. Kotliar and A. Aligia for useful discussions.
[1] A. Georges, G. Kotliar, W. Krauth, and M. J. Rozenberg, Rev. Mod. Phys. 68, 13 (1996).

[2] A. Georges and G. Kotliar, Phys. Rev. B 45, 6479 (1992).

[3] W. Metzner and D. Vollhardt, Phys. Rev. Lett. 62, 324 (1989).

[4] C-H. Yee, G. Kotliar, and K. Haule, Phys. Rev. B 81, 035105 (2010).

[5] A. B. Shick, L. Havela, A. I. Lichtenstein, and M. I. Katsnelson, Sci. Rep. 5, 15429 (2015).

[6] K. Haule, C.-H. Yee, and K. Kim, Phys. Rev. B 81, 195107 (2010).

[7] K. Haule, J. H. Shim, and G. Kotliar, Phys. Rev. Lett. 100, 226402 (2008).

[8] X. Deng, K. M. Stadler, K. Haule, A. Weichselbaum, J. von Delft, and G. Kotliar, Nat. Commun. 10, 2721 (2019).

[9] D. J. García, K. Hallberg, and M. J. Rozenberg, Phys. Rev. Lett. 93, 246403 (2004).
[10] K. Hallberg, D. García, P. Cornaglia, J. Facio, and Y. NúñezFernández, Europhys. Lett. 112, 17001 (2015).

[11] Y. Núñez- Fernández and K. Hallberg, Front. Phys. 6, 13 (2018).

[12] F. A. Wolf, I. P. McCulloch, O. Parcollet, and U. Schollwöck, Phys. Rev. B 90, 115124 (2014).

[13] M. Ganahl, P. Thunström, F. Verstraete, K. Held, and H. G. Evertz, Phys. Rev. B 90, 045144 (2014).

[14] D. Bauernfeind, M. Zingl, R. Triebl, M. Aichhorn, and H. G. Evertz, Phys. Rev. X 7, 031013 (2017).

[15] Y. Núñez- Fernández, G. Kotliar, and K. Hallberg, Phys. Rev. B 97, 121113(R) (2018).

[16] Y. Komijani, K. Hallberg, and G. Kotliar, Phys. Rev. B 99, 125150 (2019).

[17] F. B. Kugler, S-S. B. Lee, A. Weichselbaum, G. Kotliar, and J. von Delft, Phys. Rev. B 100, 115159 (2019).

[18] Y. Niu, J. Sun, Y. Ni, J. Liu, Y. Song, and S. Feng, Phys. Rev. B 100, 075158 (2019). 
[19] J. Rincón, E. Dagotto, and A. E. Feiguin, Phys. Rev. B 97, 235104 (2018).

[20] M. Karski, C. Raas, and G. S. Uhrig, Phys. Rev. B 72, 113110 (2005).

[21] S-S. B. Lee, J. von Delft, and A. Weichselbaum, Phys. Rev. Lett. 119, 236402 (2017).
[22] J. Kanamori, Prog. Theor. Phys. 30, 275 (1963).

[23] A. Georges, L. deÕ Medici, and J. Mravlje, Annu. Rev. Condens. Matter Phys. 4, 137 (2013).

[24] S. White, Phys. Rev. Lett. 69, 2863 (1992).

[25] K. Hallberg, Adv. Phys. 55, 477 (2006). 